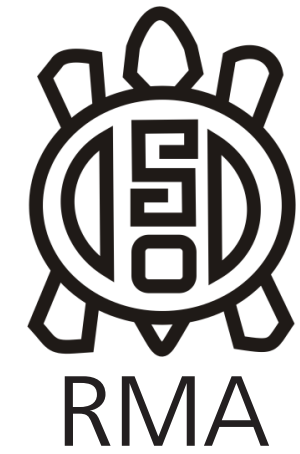

Antropología Social

\title{
El libro de los secretos de la agricultura del Fray Agustín y las relaciones humano-plantas durante la modernidad temprana
}

\author{
Fray Agustin The Book of Agricultural Secrets and the Human-Plants \\ Relationships during Early Modernity
}

*Instituto Argentino de Nivología, Glaciología y Ciencias Ambientales (IANIGLA), CCT-CONICET Mendoza. Ruiz Leal s/n Parque General San Martín, Mendoza. Instituto de Arqueología y Etnología Salvador Canals Frau, FFyL-UNCuyo.

E-mail: luismafferra@gmail.com

\begin{abstract}
Resumen
A partir del análisis de un libro de agricultura escrito en el año 1617 en España, propongo discutir los conceptos utilizados por las ciencias antropológicas para comprender las vinculaciones de la gente con las plantas, en especial con los árboles, en la modernidad temprana. Por medio de un ejercicio comparativo reflexiono sobre la aplicación de los dualismos modernos sujeto/objeto y silvestre/doméstico. Observo algunos matices que reconfiguran aquí estas categorías, en el primer caso, dados por el reconocimiento de analogías entre la anatomía vegetal y humana, así como de cierta sensibilidad y sociabilidad en los árboles. En el segundo, basándose en la no taxativa distinción de las plantas, las prácticas, los ámbitos y las valoraciones asociadas a lo silvestre y lo doméstico. Además, analizo el entramado relacional en el que se incluyen los árboles en el caso estudiado, el cual permite observar órdenes temporales y espaciales, así como la afectación de estos sobre la materialidad. En suma, el trabajo apunta a problematizar el esquema relacional que vinculó a humanos y árboles en la práctica agrícola durante la modernidad temprana, los resultados obtenidos son de interés comparativo tanto para España y Europa occidental como para otros contextos asociados a su expansión colonial.
\end{abstract}

Palabras Clave: Árboles, Sujeto-objeto, Silvestre-doméstico, Giro ontológico, Período Colonial

\begin{abstract}
Based on the analysis of an agriculture book written in 1617 in Spain, I propose to discuss the concepts used by the anthropological sciences to understand people relationships with plants, especially with trees, in early modernity. Through a comparative exercise, I reflect on the application of modern subject/object and wild/domestic dualisms. I observe some nuances that reconfigure these categories here, in the first case, given by the recognition of analogies between plant and human anatomy, as well as some sensitivity and sociability in trees. In the second, based on the non-taxative distinction of plants, practices, places and valuations associated the wild and domestic. In addition, I also analyse the relational framework in which trees are included in the studied case, which allows me to observe temporal and spatial orders as well as their affectation on materiality. In short, the work aims to problematize the relational scheme that linked humans and trees in agricultural practice during early modernity. The results are of comparative interest both for Spain and Western Europe and for other contexts associated with its colonial expansion.
\end{abstract}

Keywords: Trees, Subject/object, Wild/domestic, Ontological turn, Colonial Period.

En este trabajo analizo el Libro de los secretos de la agricultura, casa de campo y pastoril publicado por primera vez en idioma catalán en 1617 por el Fray Miguel Agustín (1560-1630) en Cataluña, España. Esta primera versión contenía tres libros o partes; en 1626, cuando se publicó en castellano, se le añadieron otras dos partes que completaron el estado definitivo de la obra'. El análisis textual de Luis Núñez (2008) muestra que en lo fundamental su contenido es similar al de tratados previos, como por ejemplo Agricultura general

\footnotetext{
1 En este caso analizo una versión en castellano editada en 1722, impresa por Juan Piferrer, original conservado en Biblioteca Catalunya (ubicación: R (6) -8-52), publicado por Alacant: Biblioteca Virtual Miguel de Cervantes, 2002.
} 
del campo de Alonso de Herrera, libro que también observo para apuntalar algunos aspectos ${ }^{2}$. Éste fue publicado originalmente en 1513 y fue el primer tratado de agricultura escrito en una lengua romance y que incorporó además conocimientos de autores árabes (Quiroz García, 2015; Willis, 2007). ${ }^{3}$ Volviendo al libro de Agustín, se afirma que su contenido es también muy similar al de L'agriculture et maison rustique publicado en 1570 en París por Charles Estienne y Jean Liébaut. Sin embargo, el Libro de los secretos de la agricultura logró mayor vigencia que sus antecesores, posiblemente debido a que presentaba la información de una forma más clara y concisa, así como agregaba instrucciones prácticas y disertaciones morales, elementos que tal vez propiciaron su continua reedición, especialmente durante el siglo XVIII (Núñez, 2008, pp. 201-204).

Las particularidades señaladas hasta aquí, hacen que el libro de Agustín sea interesante para analizarlo desde una perspectiva antropológica. De este modo, si bien tanto este autor como los otros mencionados citan autores clásicos (por ejemplo, a Catón, Cicerón o Columela), adaptan los contenidos de estos ensayistas latinos a los saberes locales y a los sentidos del siglo XVI y XVII (Willis, 2007). Además, Agustín afirma conocer los secretos y tener experiencia directa en las labores campestres. Así también, es común hallar en estas obras modernas referencias sobre la opinión de actores anónimos que formaron parte del contexto en el que fueron escritas, por caso Agustín menciona continuamente lo que "otros" prefieren y opinan, así como Herrera refiere a conocimientos sostenidos "según los agricultores". Todo esto permite analizar la información contenida utilizando las herramientas analíticas de la antropología histórica (Viazzo, 2003), y proponer así interpretar los sentidos y categorías que conforman la perspectiva de los actores estudiados. Si bien la obra de Agustín ya ha sido analizada por especialistas de diferentes disciplinas científicas que buscaron informar sobre el desarrollo de algún conocimiento específico, tal como el manejo de frutales (Castro, 2015), la parasitología animal (Cordero del Campiño y Rojo Vázquez, 1979) o la arquitectura (Serra i Clota, 2011), en este caso, más que los conocimientos concretos, me importa la forma en la que éstos fueron esquematizados, en especial, en función de categorías de relación entre personas y plantas y fundamentalmente para el ámbito práctico de vida cotidiana y la agricultura. Estimo que al respecto tanto la obra de Agustín como la de Herrera tienen gran valor informativo.

En concreto, mi interés en esta materia deriva del análisis de restos arqueológicos de carbón vegetal asociados

\footnotetext{
2 Analizo puntualmente una copia impresa en 1615, por Domingo González y conservada en la Universidad de Granada (ubicación: A-46203).

${ }^{3}$ Si bien estos autores no fueron citados directamente, se estima que tal omisión se deba al riesgo que suponía referenciar fuentes que eran profanas para la España católica (Willis, 2007).
}

al periodo colonial americano (siglos XVI a XVIII), por lo que debo advertir que mis preguntas fueron motivadas por los problemas asociados a este campo de conocimiento. En lo puntual, al intentar diferenciar modos de relación entre sociedades humanas y árboles (Mafferra, 2018), no hallé contribuciones que propongan categorías de análisis para dicho contexto de estudio. Por el contrario, las interpretaciones comúnmente construidas no problematizan el uso análogo de las categorías modernas de vinculación entre sociedad humana y ambiente (Marconetto y Mafferra, 2016). Sin embargo ¿las categorías de relación humano-vegetales en la modernidad temprana fueron las mismas que las actuales? En busca de generar un aporte a este problema, propongo evaluar de modo heurístico la vigencia de dos dualismos subsidiarios del de naturaleza/ cultura y que han sido profusamente utilizados en las ciencias antropológicas, sobre todo en la arqueología: el de sujeto/objeto y el de silvestre/doméstico. Para ello, identifico sentidos o relaciones que no encajan dentro de la modernidad, así como otros indicios que permitan analizar las comprensiones relativas al caso de estudio (Ginzburg, 1994; Levi, 1996). Busco también remontar el ensamble de relaciones en el que se incluyen las plantas en este contexto (Latour, 2008), lo que posibilita discutir órdenes espaciales y temporales. De esta manera, mis objetivos para este trabajo se acercan a la agenda de la historia cultural y no así a la historia de la ciencia. ${ }^{4}$ Es decir, no me centro en la filosofía natural y el desarrollo científico temprano dados durante el periodo de estudio, ya que entiendo que las obras analizadas se compusieron al margen de estos campos 5 .

En suma, reflexionaré sobre las relaciones humanoplantas, en especial, humano-árboles. Comenzaré discutiendo la vigencia de las categorías modernas sujeto/ objeto, luego haré lo propio con el par silvestre/doméstico, para posteriormente analizar el entramado asociativo en el que se incluyen las plantas en el contexto de estudio. Finalmente, retomaré las principales conclusiones y analizaré su alcance en relación a la pregunta que motiva este trabajo. Los resultados obtenidos me permiten discutir aspectos inherentes a la materialidad, sociabilidad

\footnotetext{
${ }^{4}$ Un buen referente de la historia cultural de las plantas es Pastoureau (2013); otros trabajos interesantes, aunque con un nivel interpretativo menos profundo, son los de Musselman (2003) o Prance y Nesbitt (2005). Sin embargo, este campo no está tan desarrollado como otros con los que podría compararse, tal como la historia cultural de los animales (Morgado García, 2011; Pastoureau, 2008, 2015; Salisbury, 2012). Para el caso de las plantas, son más abundantes los trabajos de orientación cultural concentrados en fenómenos particulares tales como la comida, el consumo o uso medicinal (Dillinger et al., 2000; Gately, 2003; Mintz, 1996).

5 Durante los siglos XVI y XVII la ciencia alineó su interés con la expansión colonial, lo que fomentó especialmente el desarrollo de las áreas de conocimiento cosmográfico y náutico, y dirigió sus objetivos al descubrimiento del mundo natural de las Indias, aspecto que se revertiría recién durante la llustración (Bleichmar et al., 2009; Nieto Olarte, 2009; Piñero, 1979; Puerto Sarmiento, 2009; Salavert Fabiani, 1995; Sánchez Martínez, 2011, 2014).
} 
y agencia de los árboles, como así también categorías espaciales y temporales relativas a las plantas y a la práctica agrícola.

\section{La carne y el alma de los árboles}

Comenzaré abordando a los árboles como objetos. Desde la perspectiva moderna posterior a los aportes de Linneo en el siglo XVIII, plantas y humanos pertenecen a reinos diferentes; se trata de seres cuyas fisicalidades se encuentran distinguidas de forma clara. ${ }^{6}$ No obstante, en tratados más antiguos esta diferenciación no es tan evidente, por caso, en El libro de los secretos de la agricultura hallé algunos indicios que permiten entender el funcionamiento de la vida vegetal, en especial el de los árboles, conforme al funcionamiento de la vida humana. Entre estos, los métodos para el cuidado de las plantas son significativos. De este modo, al igual que en las personas donde el sangrado permite curar fiebres o infecciones, entre los árboles el uso de barrenos para perforar el tronco posibilita extraer sustancias nocivas y así sanar los frutos corrompidos de guindos y cerezos, o endulzar las naranjas en una planta que las da agrias (Agustín, 1722, pp. 106, 112). Aquí, árboles y humanos (y también algunos animales, por cierto), se curan con tratamientos análogos con base en la teoría humoral. Ésta, desde la Antigüedad al siglo XIX, explicó el funcionamiento del cuerpo humano (Hayward, 1989), pero sirvió además para entender la fisiología de muchas clases de seres, entre ellos las plantas (Orlob, 1971; Volcy, 2007).

En el mismo sentido, en la obra de Agustín (1722) hallé algunas analogías al cuerpo humano o animal para explicar la fisiología de los árboles. El autor describe el corazón -refiriéndose a la médula- como "la parte más humeda, y más vital"7 que da alimento al árbol y a la fruta "baxando, y fubiendo por los troncos" (p. 134). Por ello, algunos árboles como los sauces o álamos por tener "grande corazon ... producen muchos troncos, y leña" o "fe plantan fin raíces" (pp. 139, 165). En el material de estudio, la presencia de estas comparaciones parece continuar un esquema que caracterizó al pensamiento antiguo, medieval y renacentista, donde el razonamiento analógico vinculaba seres en base a la comparación de

\footnotetext{
${ }^{6}$ Utilizo los conceptos de fisicalidad e interioridad siguiendo a Descola (2012, pp. 182-183), quien los propone para analizar la distinción entre los componentes materiales e inmateriales de la persona. El autor afirma que, si bien dicha diferenciación es universal para los casos registrados por la historia y la etnografía, se observan diversas maneras de objetivarla, por lo que es preciso enunciar categorías que no proyecten la oposición occidental entre espíritu y cuerpo. Así, la fisicalidad alude al conjunto de las expresiones visibles y tangibles resultantes de las características materiales, fisiológicas o morfológicas de los cuerpos orgánicos e inorgánicos; y la interioridad implica una gama de propiedades inmateriales reconocidas en los seres humanos, es decir, intencionalidad, subjetividad, reflexividad, afectos, actitud para significar, principios de acción o animación, etc.

7 Las citas textuales de los autores antiguos conservan la ortografía original.
}

estructuras y especialmente de funciones (Ferigolo, 2014). Así, del mismo modo que actualmente se distinguen a los animales y seres humanos de los árboles, en los tratados medievales esta diferenciación no es tan tajante (Sodigne-Costes, 1991), dando lugar al establecimiento de semejanzas como la del corazón y la médula. Estas analogías no fueron anuladas durante el Renacimiento, sino por el contrario reforzadas, por ejemplo, la vieja equivalencia entre plantas y animales, donde la planta era un animal parado de cabeza, con su boca -o sea, la raíz-undida en la tierra, es ahora dotada de nuevos argumentos. De esta manera, la semejanza desempeño un rol crucial en el modo de componer el mundo en Occidente, por lo menos hasta comienzos del siglo XVII (Foucault, 1968, pp. 26-30), habilitando modos de relación que me interesa discutir.

Si bien el reconocimiento de semejanzas entre la anatomofisiología en distintos órdenes de seres es una forma de explicación que no se restringe al caso abordado, sino que es muy común en los modos de conocer de sociedades muy diversas. ${ }^{8}$ En el caso medieval y renacentista, es significativa la correspondencia entre árboles y personas (Pastoureau, 2013, pp. 90-91). Concretamente, se propone que el árbol es antropomorfo, así la madera y la carne, la corteza y la piel resultan análogas, por lo que estas plantas pueden tanto como los humanos sufrir, podrirse, ser heridas o infectadas por gusanos. Es sugestivo como esta humanización del árbol afecta su materialidad, sacando a la madera del lugar inerte dado a otros materiales y también condicionando la consideración que se tiene de los oficios relativos a ésta. Por ejemplo, los leñadores y carboneros como enemigos de los árboles son ligados al mal y al demonio, es gente corrompida por su oficio. Sin embargo, los artesanos del rubro tienen la más alta consideración, trabajan con la materia prima por excelencia, viva y pura, despierta el respeto y el apego (Le Goff, 1999, pp. 178-182; Pastoureau, 2013, pp. 92-93).

Es interesante como ciertas características en las fisicalidades de los árboles los acercan a la humanidad, lo que hace dificultoso pensarlos como objetos sin más. Por lo tanto, para continuar el juego de categorías propuesto, resultará relevante cuestionar si podrán ser en cambio sujetos. En tal caso, la dualidad moderna sujeto/ objeto se caracteriza por reservar para la humanidad una interioridad particular, que deja al resto de los seres y cosas, incluidas las plantas, en un rol pasivo o mecánico (Jones y Cloke, 2008). Resulta ilustrativa la cita de un libro de historia natural del siglo XIX donde es la fisicalidad de las plantas la que determina su rol de objetos cuasiinanimados, ya que son seres "cuya vida se halla reducida a la nutrición y reproducción, sin nada de sensibilidad ni motilidad, ejecutan todas sus funciones de un modo tan fijo e invariable, que no poseen ningún dominio sobre su

\footnotetext{
${ }^{8}$ Por ejemplo, se observa en los canacos de Melanesia (Leenhart, 1961) o en los anewene-nawes de la Amazonia (Mendes Dos Santos, 2003).
} 
propia existencia" (Yáñez y Girona, 1845, p. 5).

En la modernidad, esta diferenciación se asienta en la exclusiva posesión por parte de los humanos de alma, espíritu o mente. Desde la antigüedad clásica, se entendía que las plantas poseían un alma vegetativa, que propiciaba el crecimiento y era compartida por todos los seres vivos. Sin embargo, se diferenciaba de la de otros seres como los animales que tenían también un alma sensible, y ambas, de la de los humanos, que además de crecer y sentir podía razonar o pensar (Ferigolo, 2014). Estas concepciones fueron mantenidas en el campo erudito durante la Edad Media y el Renacimiento (Sodigne-Costes, 1991), sin embargo, ¿cuál era la comprensión por fuera de estos ámbitos? En relación con la práctica agrícola, un primer indicio que revela la ocurrencia de fenómenos diferentes es dado cuando Agustín o Herrera refieren continuamente en su libro a lo que las plantas "quieren", "defean", "aman" u "odian". Así, algunas plantas como el arrayán temen al frío, otras como el laurel aman estar a orillas del mar, o los cítricos se recrean con los aires del mediodía (Agustín, 1722, pp. 106-109; Herrera, 1615, p. 75b). Estas demostraciones de sensibilidad escapan a la conceptualización que repasamos más arriba y hacen presumible que en este contexto las plantas podían poseer una interioridad no radicalmente diferente a la de los humanos, o por lo menos asimilable a la de los animales.

Podría objetarse que dichas alusiones pueden entenderse como simples figuraciones; sin-embargo, la diferencia en las conceptualizaciones vigentes en este contexto es notoria al observar cómo entre los árboles existían afinidades sociales variables. Es decir, no todas las plantas se llevaban bien con todas, por lo que Agustín (1722) se esmera en detallar un orden de sociabilidad. En éste, el laurel es enemigo de las vides, pero buen amigo del cerezo. Por su parte, el olivo es buen amigo de la higuera, junto a la que se lo ve muy alegre, más no así de la encina, por quien tiene un rechazo mortal (p. 109). Algunos árboles son auténticamente odiosos, entre ellos destacan los nogales, muy celosos "aborrece en eftremo la hermandad, y compañía de otro arbol" (p. 125).

Este fenómeno puede entenderse nuevamente ordenado por las semejanzas, más precisamente dentro del juego entre las simpatías que afecta el orden de mundo renacentista (Foucault, 1968, pp. 32-34). La simpatía atrae a las cosas en función de sus propiedades e incluye el peligro de asimilar; es compensada igualmente por su figura gemela y contraria, la antipatía, que hace que las cosas se repelan. Estas atracciones o rechazos suelen fundamentarse en la naturaleza material del mundo, es decir, en las cualidades humorales que componen las cosas, tema que trataré más adelante. Sin embargo, la obra de Agustín contiene indicios que indican la posible existencia de relaciones de naturaleza diferente, que no se explican únicamente desde plano material. Por ejemplo, es evidente en el hecho de que estos conflictos entre plantas se presentan como históricamente construidos, por caso, dice Agustín (1722) que entre nogales y encinas existe "una enemiftad muy antígua" (p. 125). Por otro lado, estas afinidades no se expresan de forma pasiva, sino que suponen respuestas, cuyas consecuencias eran de interés para el agricultor. Así, el autor agrega, por ejemplo, que la sombra de los nogales "hace gran daño a todos los demás árboles que tiene vecinos", incluso a los de su propia especie, por lo que "quiere grande efpacio de tierra, el uno del otro, por lo menos quarenta pies, porque la fombra del uno no dañe al otro" (p. 125). A la vez, otras fuentes de este período aportan como sus raíces - de una extensión maravillosa- estropean la tierra e impiden el crecimiento de otras plantas (Estienne y Liebault, 1689, p. 333). Este tipo de reacciones entre plantas puede constatarse de manera empírica y es entendida actualmente dentro del marco de la aleopatía ${ }^{9}$, de la cual, los tratados mencionados son considerados antecedentes (Willis, 2007). Sin embargo, en las obras antiguas, la influencia negativa de los árboles perniciosos no era sólo capaz de afectar a otras plantas, sino también de enfermar a las personas, en especial con dolores de espalda y cabeza (Herrera, 1619, p. 85), e incluso matar animales domésticos (Pastoureau, 2013, p. 105). Por su parte, así como la sombra del nogal puede dañar a otras plantas y hierbas, también menciona Agustín (1722) como "quiere fer limpiado á menudo por todo fu contorno" ya que si se dejan hierbas "hacen venir las nueces vacias" ( $p$. 125). Esto último hace presumible que el árbol no solo tiene la capacidad de otorgar respuestas ante ciertos estímulos, dañando plantas, animales o personas, lo que se equipararía en este contexto a una sensibilidad animal, sino que vale al menos para preguntar si la capacidad de dirigir esa respuesta para afectar directamente al interés humano de la relación, es decir, el beneficio de los frutos, ¿era parte de un comportamiento racional? Es decir, además de la aparente sensibilidad que demuestran las plantas ¿está dotado el nogal de una interioridad asimilable a la humana?

Los materiales analizados hasta aquí sirven para cuestionar la vigencia del dualismo moderno sujeto/ objeto para el caso de los árboles. La comprensión repasada supone que estas plantas poseen una anatomía en algunos aspectos análoga a la animal o humana. Además, los árboles se describen como dotados de cierta sensibilidad y de una presumible interioridad asimilable a la humana, caracterizada por la existencia de simpatías y antipatías entre las plantas, que eran cuando menos dentro del discurso del libro de Agustín -así como en otros de la época-, representadas a modo de un orden de sociabilidad, literalmente amistades o enemistades. Esta socialización de la naturaleza durante la modernidad temprana sería coherente con una continuidad de las comprensiones animistas medievales, que no solo

\footnotetext{
9 A grandes rasgos, la alelopatía se entiende actualmente como el efecto nocivo que una planta tiene en otra debido a los químicos que libera al medio ambiente (Willis, 2007).
} 
humanizaron a los árboles sino que consideraron de manera general al ambiente como sujeto. Se propone que estas concepciones habrían persistido en la cultura popular europea incluso hasta bien entrado el siglo $\mathrm{XX}$ (Barros, 1997).

\section{Lo silvestre y lo doméstico: Las formas, las prácticas, los ámbitos y las jerarquías}

La comprensión moderna de la relación humano-planta prevé otro dualismo que deseo discutir en el marco de la obra del Fray Agustín. Este es el dado entre lo silvestre y lo doméstico, e implica cuatro aspectos que considero problemáticos. Primero, la domesticación de una planta involucra cambios morfológicos detectables que la hacen dependiente de la acción humana. Segundo, supone una distinción del tipo de relaciones y prácticas dadas en torno a las plantas correspondientes a cada categoría. Tercero, diferencia con claridad ámbitos para cada una de ellas. Y cuarto, implica una jerarquización valorativa de lo doméstico por sobre lo silvestre.

En cuanto al primero de los aspectos señalados, en la obra de Agustín se distinguen las plantas domésticas de las silvestres. Dicha diferenciación es dada por la posibilidad que tienen algunas especies para fructificar más y con mejor calidad y belleza, es decir, la morfología de los frutos parece ser un aspecto fundamental en esta distinción. En relación con los árboles, es interesante cómo esto se logra a partir de la acción humana, en base sin embargo a la conjugación de elementos tanto silvestres como domésticos, puntualmente, a partir del arte de los injertos. Con este, explica Agustín (1722), se consigue que los árboles silvestres se hagan domésticos, dando así frutas más "bellas, hermofas y mejores" ( $p$. 146). De este modo, lo silvestre se asocia aquí a lo estéril y lo doméstico a lo fértil. Sin embargo, el autor aconseja siempre injertar encima de un árbol silvestre "que es durable, y de más robufto natural" (p. 146). Lo ideal es asociar plantas de la misma especie o, en su defecto, árboles del mismo tipo de semilla o carozo, o entre plantas con similares temporalidades, las tardías pueden injertarse con las tardías o las tempranas con las tempranas. Otro aspecto a tener en cuenta es la similitud en la dureza de su madera o de las gomas o aceites contenidos por ésta (Agustín, 1722, pp. 146-147). Para mayor precisión, para cada árbol se explica su posibilidad para ser injertado con otras especies según su grado de hermandad. En este juega también un papel especial el orden de simpatías arriba señalado. Entonces, la planta doméstica es la que produce frutos de mejor calidad $y$, para el caso de los árboles, la acción humana es la que conjuga los elementos en pos de este fin. Dicha práctica combinaba igualmente en una misma planta, una base o pie silvestre, asociado a lo durable y robusto, con tallos de variedades con frutos más valorados. Esta práctica debía completarse también con la de la agricultura, ya que estos árboles "fino los labran, limpian, y beneficiam, no darán fruto, antes bien se bolverán efteriles" (Agustín, 1722, p. 140). Aquí la planta doméstica es producida "en relación" y no producto "de una relación", esto difiere de la idea de dependencia planteada en la modernidad y supone que tanto plantas como agricultores se reproducen en esta asociación. Sólo el sostenimiento de la agricultura hace a lo domestico estable, por lo que se trata de una condición reversible.

En segundo lugar, con respecto a la distinción -dentro de la comprensión moderna- del tipo de relaciones dadas en torno a las plantas domésticas o silvestres, observé que en el libro de Agustín el árbol doméstico es producido en relación con las prácticas humanas. Así también, en la obra puede distinguirse una segregación de los espacios, a grandes rasgos, se menciona que las plantas deben cultivarse dentro del huerto (los cultivos en torno a las casas) o fuera de éste (en los contornos del huerto, o en campos de cultivo ubicados por fuera del ámbito doméstico) o en el bosque. Sin embargo, tal distinción entre las plantas y tal distribución espacial no implica formas de vinculación diferentes. Así, Agustín (1722, p. 167) propone para todas las plantas un mismo tipo de trato: la agricultura. Si bien esta práctica se suele analizar asociada a las plantas y ámbitos domésticos, aquí se hace tanto en relación con las plantas del huerto, que dan frutos comestibles, como con las del bosque, que dan leña para quemar. De este modo, la agricultura es considerada un modo general de relación de los humanos con las plantas. El autor aclara que todas las especies la necesitan, o bien, casi todas, exceptuando algunas hierbas o malezas que "le temen, como por fu grande enemiga" (p. 168). Esto hace previsible que se trate entonces de un modo de relación general, en tanto resulte productiva, contando la excepción del algarrobo ${ }^{10}$, que si bien quiere ser plantado, injertado y regado no se "debe fenbrar, porque no haría fruto, y peligraría de morisfe prefto" (p. 140).

En cuanto al tercer aspecto planteado, referente a la distinción moderna entre los ámbitos asociados a lo silvestre y lo doméstico. Como dije, Agustín indica una distribución espacial de los árboles ya sea en el huerto, fuera de éste o en el bosque (Tabla 1). Sin embargo, el orden al que responde esta distribución no parece estar determinado por la condición doméstica o silvestre de las plantas que integran cada ámbito, sino por otros fenómenos. Para empezar, algunos árboles deben estar en el huerto simplemente porque son lindos. Entre ellos, Agustín (1722) explica que el arrayán debe plantarse "en el huerto, y en algún lugar viftoso, porque es un árbol muy apacible, y de grande recreo" (p. 108). Del mismo modo, la palma debe estar en el huerto por su "belleza, y agradable vifta" o el laurel por su "verdor, y belleza que tiene todo el año" (p. 108-109). Así también, la ubicación de los árboles en el huerto debe tener en cuenta las afinidades sociales entre plantas que repasamos más

\footnotetext{
10 En referencia a Ceratonia siliqua, planta originaria de la cuenca del Mediterráneo.
} 
arriba. En este sentido, en su "Agricultura general del campo" Herrera (1619, p. 53b) explica que al igual que los humanos se distribuyen en un poblado, en base a su oficio o religión, de tal forma que los oficios sucios o viles no se mezclen con los limpios y virtuosos, o como los cristianos y los árabes no deben vivir juntos, las plantas deben separarse entre sí en base a sus características para evitar problemas. Por lo que las amistades y enemistades señaladas más arriba deben tenerse en cuenta para comprender el orden espacial de las plantas en este contexto. En relación con esto, otro aspecto reiteradamente mencionado refiere a que tal o cual especie debe estar en tal o cual lugar porque es donde se siente más a gusto. Esto se lograba respetando el orden de compañías deseables y evitando las indeseables, y también eligiendo suelos, lugares o contextos, así como temperaturas, vientos y correspondencias astronómicas indicadas para cada especie. Por caso, "quiere el granado la tierra barrofa, y poco gorda; plantafe en el mes de marzo, y abril, en lugar caliente" de preferencia "á la parte del Medio Dia, y no al Levante, ni Poniente, que efte arbol quiere estár al contrario de los otros; y no quiere la tierra efteril, ni húmeda". También "hacefe mucho mejor plantándose muy cerca de la cebolla albarrana" que por su "contrariedad natural impedirán que dichas granadas no fe abran". Además, es amigo del arrayán, pero "el naranjo, falvia, y morál, no fon amigos". Este árbol "padece" la niebla, por lo que debe ser plantado cerca de una pared o casa a fin de que no reciba con tanta facilidad este humor que le es dañoso (Agustín, 1722, pp. 110-111). Este conjunto de consideraciones servía para distinguir cuál era el lugar indicado de los diferentes árboles, pero implicaba que tanto dentro como fuera del huerto se plantasen indistintamente especies que son para nosotros silvestres o domésticas. Por ejemplo, en la obra de Agustín (1722) se evidencia que especies domésticas como los naranjos o durazneros se dan mejor en el huerto, pero los olivos o nogales crecen mejor fuera de éste (pp. 105, 122). O bien, árboles que actualmente pueden considerarse silvestres como el arrayán o los cipreses se dan mejor dentro del huerto (pp. 108, 110), como también algunos frutales pueden ir tanto en el huerto como en el bosque (p. 166).

Con respecto al cuarto y último aspecto señalado al comienzo de esta sección, la jerarquización dada desde la perspectiva moderna de lo doméstico por sobre lo silvestre, enlaza los ámbitos y prácticas del primero con pueblos más desarrollados y, por contraste, relaciona los del segundo con los menos avanzados. Dicha asimetría no es fácilmente distinguible en la obra de Agustín. Ya señalé cómo la agricultura parece ser un modo de relación general entre humanos y plantas, por lo que plantas y ámbitos no estarían diferenciados en base a ésta. Por lo mismo, los dos ámbitos son construidos en relación a la acción humana; Agustín menciona que los bosques "se hacen" al igual que los huertos. Así, para cada uno de estos espacios el autor dedica por igual un capítulo completo, mostrando que no habrían existido diferencias de jerarquía entre estos ámbitos. Herrera (1619, pp. 47-97) por su parte, presenta en un mismo libro, juntos e intercalados árboles de ambas clases. Otro aspecto interesante es que para Agustín (1722) los bosques pueden hacerse tanto con árboles para nosotros silvestres como domésticos, priorizando aquellos que además de dar leña y madera también fructifican, como la encina o el castaño. Así también, si bien los pinos hacen en los montes altos "bofques de por sí", esto no implica que otros bosques puedan hacerse plantándolos en "tierra muy bien arada" (p. 165). Una vez establecidos, no parece haber distinción entre unos y otros, ya que se presentan como bosques por igual. Ambos deben cultivarse al menos dos veces al año, ya que mientras más los cultiven serán mejores y más frondosos. Dicho cultivo consiste en general en el riego y desmalezado, sobre todo de las plantas jóvenes (pp. 162-167). Incluso las prácticas que podrían considerarse como meramente extractivas, como el corte de leña o madera, también se presentan asociadas a la acción de asegurar un crecimiento "muy prefto" de los árboles del monte o el bosque (p. 16). Así, éstos últimos no fueron valorados de forma negativa frente a los huertos, por lo que no existen a priori jerarquizaciones en torno a los ámbitos o las plantas presentes en cada caso. Igualmente, debe de considerarse lo aportado por otros autores que argumentan una coexistencia ambigua entre naturaleza y cultura esquematizada por la cultura europea (Hell, 2001, p. 238), pudiendo el ambiente tornarse un espacio contradictoriamente paradisíaco o aprensivo (Nash, 2014, pp. 8-9); en relación a la herencia medieval, el bosque es señalado como el arquetipo del lugar natural en la Edad Media, resultando un espacio al mismo tiempo tan proveedor como temido (Le Goff, 1986, p. 32).

\section{Los árboles y el cosmos}

En este apartado propongo analizar y reensamblar el complejo entramado asociativo en el que se encontraban inmersas las plantas en la obra de Agustín. El colectivo resultante puede relacionarse con la composición de mundo asociada al periodo medieval-renacentista y supone una mixtura de esencias, formas y sustancias. Esta heterogénea multiplicidad se recomponía en una densa red de analogías que vinculaban las propiedades intrínsecas de las entidades distinguidas. Es decir, las cualidades, movimientos o modificaciones en algunas cosas o seres afectaban a otros de forma analógica (Descola, 2012, p. 301). Foucault (1968, pp. 26-34), propone que estas asociaciones podían ordenarse en relación con cuatro formas: la convenientia o vecindad, que asemeja lo cercano, la aemulatio o emulación de cosas distantes (tanto como de un punto a otro universo), la analogía, que superpone las dos anteriores pero no refiere a los ajustes más visibles, sino a las relaciones más sutiles, y finalmente la simpatía, que atrae o repele a las cosas y que vimos daba lugar a la amistad o el odio entre 
ciertas plantas. En este sentido, me interesa en especial revistar como esta comprensión promueve asociaciones no previstas desde nuestra perspectiva.

Para empezar, la obra del Fray Agustín (1722) está repleta de afirmaciones que permiten reconocer la vinculación de fenómenos relativos al macro y microcosmos, recurrencia que indica que se trata de un aspecto gravitante en la comprensión del manejo agrícola. De ello, resulta ilustrativo también una "Rueda perpetua" incluida al final del libro, útil para identificar en base a preceptos cosmológicos los años fértiles e infértiles. Del mismo modo, a lo largo de la obra se detallan prácticas que procuran "encaminar" la vida vegetal tras la dinámica de los astros, ya que plantas y árboles crecen por su "vital infpiración, y movimiento" (p. 8). Estas emulaciones y analogías daban lugar a una ordenación de la temporalidad, indicando con precisión diferentes prácticas agrícolas correspondientes a las cuatro estaciones y a cada uno de los meses del año.

Así, Agustín (1722) afirma que el sol, por su "calor natural, influencia, acción y radiación celeste", da a las "cosas terreftres, ciertas facultades y virtudes" (p. 11). Por sus movimientos las "materias terreftres, fon producidas, y engendradas" en los cuatro tiempos en los que dicho astro hace su curso (p. 11). No obstante, la luna tiene mayor relevancia, por ser el "planeta" más cercano, es "madre, y governadora de todas las humedades de la tierra" ( $p$. 8), y la fuerza de su resplandor afecta el engendro de los humores en los seres vivos. Durante la luna creciente las plantas aceleran los procesos fisiológicos de crecimiento, lo que la hace deseable para la siembra o plantación, así como para el engorde de la tierra. Sin embargo, esta luna debe evitarse cuando se recogen frutos ya que acelera su descomposición. Para esto, así como para los trasplantes e injertos, la luna menguante es preferida ya que con ella retroceden los humores en las plantas (Agustín, 1722, pp. 8-11; Herrera, 1619, p. 6b).

La escala de estos órdenes no es solo anual y mensual, sino que algunas actividades deben realizarse en horarios concretos del día; por caso, para que la madera para bastimentos y edificios tenga una óptima duración debe cortarse a fin de la luna, los días más cortos del año y previo al mediodía (Agustín, 1722, pp. 9-10). Así como con la luna y el sol, otros astros como planetas e incluso cometas afectan el desarrollo de las plantas y por lo tanto son contemplados para ordenar las prácticas. Estos órdenes expanden la praxis de la labor agrícola al ajuste de fenómenos cosmológicos diversos, ya que las plantas emulan o reproducen de manera análoga el comportamiento del cosmos y, en especial, dan lugar a una temporalidad específica para este marco contextual.

Por otro lado, las relaciones de transformación por vecindad o convenientia se registran en el libro de Agustín (1722). Señalé por ejemplo cómo las granadas no se abrirán si tienen creciendo cerca cebollas, ya que

Tabla 1. Distribución espacial de especies de árboles indicada en la obra del Fray Agustín (se agrupan las especies que el autor presenta juntas).

Table 1. Spatial distribution of tree species indicated in the work of Fray Agustin (the species thet the autor presents together are grouped).

\begin{tabular}{|c|c|c|}
\hline \multirow{2}{*}{$\begin{array}{l}\text { Huerto } \\
\text { Naranjos, cidros, limones }\end{array}$} & Fuera del huerto (Campos) & Bosque \\
\hline & Olivo (contorno de los & Castaño \\
\hline Arrayán & campos) & Encina, Roble, Carrasca \\
\hline Palma & Nogal (campos de trigo) & Pino \\
\hline Laurel & Mora & Álamos blanco y negro, Sauce (cauces \\
\hline Ciprés & Azufaifo & de agua, caminos) \\
\hline Granado & Castaño & Tamarisco \\
\hline Cerezos, Guindos & Algarrobo & Sauzgatillo \\
\hline Ciruelo & Sauco (contorno del huerto) & Fresnos \\
\hline Almendro & Box & Olmos \\
\hline $\begin{array}{l}\text { Durazno, Melocotón, } \\
\text { Albaricoque }\end{array}$ & $\begin{array}{l}\text { Yedra } \\
\text { Vidalva }\end{array}$ & $\begin{array}{l}\text { Espino o Majuelos (contornos del } \\
\text { bosque) }\end{array}$ \\
\hline $\begin{array}{l}\text { Manzano } \\
\text { Membrillo } \\
\text { Peral }\end{array}$ & Retama, Cedro & $\begin{array}{l}\text { Espinos para injerir frutales (contornos } \\
\text { del bosque) } \\
\text { Nogales (contornos del bosque) }\end{array}$ \\
\hline Avellano & & Frutales en general (contornos \\
\hline Níspero & & bosque) \\
\hline Higuera & & \\
\hline
\end{tabular}


la tendencia a abrirse de unas se complementa con lo cerrado de los bulbos de la otra. También se ve en el caso de los injertos, de modo que si el naranjo se injertase sobre un árbol rústico como el "grebol" no se helará, pero su fruta no será tan dulce. En cambio, si el injerto se realizara sobre el moral, su fruta se asemejará a la de éste y será roja y dulce (p. 107); así como si el ciruelo se injertase sobre la higuera fructificará como ésta sin florecer ( $p$. 113). En otros documentos del mismo periodo pueden reconocerse la traslación de algunas características de los lugares de crecimiento de una planta a sus atributos. Por ejemplo, en una crónica del siglo XVII sobre el reino de Chile, el jesuita Ovalle (1646, p. 56) explica cómo "El guayacán se cría en los montes y cordillera y...toma de ellas lo duro y pesado...dentro de su materia, que es tanto, que parece yerro".

Otro de los aspectos distinguidos en la asociación de plantas y fenómenos diversos, es la posibilidad de reconocer "signaturas", es decir, signos o marcas visibles que revelen la ocurrencia de las relaciones ocultas entre las cosas (Foucault, 1968, pp. 34-38). Éstas son indicadas por Agustín (1722, pp. 3-7) como "señales" y permiten prever ciertos eventos, tales como fenómenos climáticos, enfermedades o cosechas buenas o malas, entre otros. Por ejemplo, si los bosques hacen ruido es señal de viento, si el trifolio recoge sus hojas revela la proximidad de la lluvia o si la encina carga mucho fruto indica que se alargará el invierno. Del mismo modo, un nogal con muchas flores y pocas hojas señala el comienzo de un año abundante, pero si a la inversa las muchas fueran las hojas y pocas las flores sería un año estéril. Por su parte, los rosales y violetas, cuando florecen y brotan en otoño señalan enfermedades. Otras plantas acoplan su comportamiento entre sí, por caso, los años en los que los almendros dan muchos frutos, el trigo también será muy fructífero y viceversa. O bien, indican fenómenos que sucederán en la sociedad humana, así, los años de muchas avellanas serán años de muchos casamientos (Agustín, 1722, p. 120).

Del mismo modo que las plantas deben adecuarse a los ciclos astronómicos, la sociedad humana se acomodaba a la vida vegetal. Especialmente en relación con los árboles pueden observarse prácticas como la labranza asociada a los ciclos semanales o mensuales, o como la poda y la cosecha asociada a ciclos anuales. También se reconocen ciclos mayores que pueden ocupar toda una vida o unir a varias generaciones humanas. De este modo, árboles longevos como la encina o el roble, que en promedio viven cerca de trescientos años, se deben plantar para el disfrute de las futuras generaciones, como otros los plantaron para nosotros. Sin embargo, Herrera (1619) aconseja no plantar árboles siendo viejo, para no dejarlos "huérfanos" (p. 47). Así, el reconocimiento de los ciclos de vida de los árboles sirve para acomodarlos a los de las generaciones humanas. Agustín (1722) sugiere un orden mediante la inclusión de un proverbio que indica
"Olivares de tu Abuelo, higueras de tu Padre y viñas de ti mifmo" (p. 196).

Por su parte, la inclusión de las plantas en asociaciones amplias y diversas es señalada además por la recurrente mención al intercambio de sustancias. De la misma manera que las flores del durazno sirven a los humanos contra la melancolía, las heces y el orín humano sirven para que los duraznos broten y no enfermen (Agustín, 1722 , p. 116). De igual modo, tal como se utilizan sustancias humanas, la cura de las plantas incluye otras sustancias animales como guano, orín, leche o miel, tanto como sustancias provenientes de otras plantas. El libro de Agustín y el de Herrera presentan cientos de ejemplos de estos intercambios que se dan indistintamente para la cura de plantas, animales o personas. La descripción de las sustancias en circulación se torna compulsiva en el desarrollo de estas obras. La complejidad de los intercambios podría comenzar a explicarse con base en la teoría humoral, ampliada a una física que incluye a todos los existentes. En donde, las sustancias contenidas o producidas por los diferentes seres conforman un orden general que dichos intercambios procuran ordenar en base a la contraposición de calidades humorales (cálido/ fresco, húmedo/seco), temperamentos (melancólico, sanguíneo, flemático y colérico), así como toda aquella cualidad o atributo físico, social o actitudinal susceptible de analogía potencial que pueda asirse por medio de su contrario. Lo mismo señala, nuevamente, la conformación y ordenación eminentemente relacional de la naturaleza de los seres y materialidades que se incluyen en estos ensambles.

\section{Los secretos de Agustín}

Propuse analizar el Libro de los secretos de la agricultura compuesto en 1617 por el Fray Miguel Agustín, buscando evaluar el uso de algunas categorías modernas para la comprensión de las relaciones entre la gente y las plantas durante la modernidad temprana. En cuanto al análisis de la aplicación del dualismo sujeto/objeto, mostré que se reconocen analogías entre las fisicalidades de árboles y humanos. A la vez, se le concede a estas plantas cierto grado de sensibilidad y se describe un particular orden de sociabilidad promovido por amistades y enemistades entre distintas especies. Todo ello, saca de plano una relación humano-planta, o al menos humano-árbol, regida por una dualidad entre sujetos humanos y objetos vegetales. Esta socialización del ambiente, evidencia una continuidad durante el periodo de análisis de las comprensiones animistas medievales.

En el caso de la dualidad entre lo silvestre y lo doméstico, las plantas de una y otra categoría se diferencian en la obra de Agustín. Lo silvestre se relacionan con la esterilidad, pero también con la robustez y resistencia, frente a lo doméstico que se distingue por la posibilidad que tienen ciertas especies de fructificar con mejor 
calidad, aunque siendo menos resistentes. Sin embargo, estas categorías no son estancas y resultan permeables e incluso complementarias especialmente para el caso de los árboles injertados. A la vez, estas plantas precisan de la agricultura para no volverse estériles, por lo que lo domestico se trata de una condición reversible. Ahora bien, las prácticas agrícolas no son reservadas únicamente a las especies consideradas domésticas, sino que suponen un modo general de relación con las plantas. Asimismo, espacios que podrían considerarse relacionados con una $u$ otra categoría (huertos, campos, bosques) no se enlazan con éstas de forma taxativa, sino que resultan también permeables. Las necesidades ambientales y cosmológicas de cada planta, la valoración estética sobre ésta, así como el orden de amistades que la afectan, son los argumentos utilizados para determinar el lugar ideal para cada especie. En el mismo sentido, no parecen existir jerarquizaciones valorativas de lo doméstico por sobre lo silvestre, sino una domesticidad extensiva a plantas y ámbitos que a priori pueden ser considerados silvestres desde la perspectiva moderna. En suma, en el caso de estudio, no se distinguen de manera radical las plantas, las prácticas, los ámbitos y las valoraciones asociadas a lo silvestre y lo doméstico. La forma amplia de entender la agricultura en este contexto es coincidente con algunos postulados relativamente recientes sobre la domesticación. Éstos, poniendo el acento en las acciones que la propician, la entienden como todas aquellas actividades de acompañamiento en el crecimiento, de manejo, sostenimiento e incluso de crianza mutua entre plantas, humanos u otras entidades (Denham et al., 2016; Ingold, 2000; Lema, 2014; Terrell et al., 2003).

Los materiales estudiados posibilitaron analizar además el entramado que vincula a las plantas con fenómenos diversos, estas asociaciones permiten observar modos de temporalidad y de afectación entre las cosas y los seres. Para el primer caso, considero el planteo de Tim Ingold (1993) para entender la temporalidad asociada a los ciclos intrínsecos de un paisaje o un modo de habitar. A partir del concepto de "resonancia" (procedente de la música orquestal), el autor propone pensarla como el ajuste de los ciclos rítmicos asociados a la vida social. En la obra de Agustín (así como en la de Herrera), el mundo vegetal emula o reproduce de manera análoga el comportamiento del cosmos, lo que da lugar a prácticas que deben "encaminar" el curso de los astros con la vida de las plantas. Asimismo, los humanos deben ajustar su vida a los ciclos vitales de los árboles. Todo ello, resulta en un modo de temporalidad donde se enlazan los ciclos cósmicos, vegetales y humanos. Este orden, se corresponde con el "tiempo natural" señalado por Le Goff (1999, p. 153) el cual, basado en la práctica agrícola, habría sido el modo de temporalidad que rigió en Occidente durante el periodo medieval.

Para el segundo caso, observé que las categorías silvestres/ domestico no determinaron los espacios de manera clara, sino más bien, los órdenes relacionales dieron paso a una forma específica de espacialidad. En esta, los ámbitos se ordenaron a razón de controlar la afectación entre los seres y las cosas. Las relaciones simpáticas, así como las emulaciones entre plantas o entre plantas y lugares fueron relevantes en este aspecto. Del mismo modo, otras prácticas buscaron mediante el intercambio de sustancias entre diferentes seres, equilibrar o componer este orden de mundo en esencia inestable.

Esto, da lugar a que la materialidad resulte en esta comprensión mucho más inestable que la prevista desde la perspectiva moderna. Por ejemplo, las posibilidades de conservación de la madera, la fruta u otros productos es afectada por la adecuación temporal de las prácticas productivas o extractivas a fenómenos astronómicos. Si bien este aspecto es el que se revela como el más relevante en dicho sentido, otros elementos como los lugares de crecimiento de algunas plantas, o la proximidad con otros seres o cosas, pueden también influir en las condiciones físicas de los materiales generados por éstas. Lo mismo, debe contemplarse para sopesar las interpretaciones funcionalistas comunes en trabajos de arqueología, que en general se fundamentan en las características de una física -estable- asociadas a un taxón, pero que debe comprenderse como inestables en este contexto.

Retomando mi pregunta inicial en la que planteé evaluar la vigencia de las categorías modernas en el contexto agrícola, durante la modernidad temprana, las reflexiones desarrolladas a lo largo de este trabajo indican una persistencia de los sentidos medievales y renacentistas en las obras de Agustín y Herrera. Esto sería coincidente con la propuesta que afirma que la revolución científica temprana de los siglos XVI y XVII no significó un impulso rupturista generalizado $u$ homogéneo para con el conocimiento occidental, sino restringido a ciertos campos, en los que incluso las continuidades fueron también muchas (Matzkevich, 2015). En lo que concierne a las plantas, también debe considerarse que durante la modernidad temprana el desarrollo de la ciencia botánica se propulsó desde el campo de la medicina y la farmacia, lo que es figurado con claridad por el hecho de que desde ese momento hasta bien entrada la ilustración, los más eminentes botánicos fueron médicos. Esto circunscribió su interés a este campo de aplicación y lo restringió de otros como el de la agricultura (Slater, 2010; Piñero y Tomás, 1996).

Para finalizar, teniendo en mente el contexto de estudio arqueológico del periodo colonial americano que me motivo a investigar los tratados agrícolas aquí analizados; este trabajo permite comprender un poco más los modos de relación humanos-plantas que habrían conformado la perspectiva colonial. En suma, las relaciones con las plantas se esquematizan por fuera del dualismo naturaleza/cultura y sus pares subsidiarios sujeto/objeto y silvestre/doméstico. Ello distingue esta comprensión 
de la perspectiva moderna, y hace que las herramientas analíticas usadas en las ciencias antropológicas deban problematizarse para trabajar con este periodo. En este sentido, espero las discusiones presentadas puedan contribuir a la construcción de categorías contextuales para interpretar la vinculación entre la gente y las plantas y para generar nuevas preguntas y problemas.

\section{Agradecimientos}

Agradezco a Verónica Lema y Bernarda Marconetto por sus valiosos comentarios a la primera versión de este manuscrito.

\section{Bibliografía}

Agustín, M. (1722). Libro de los secretos de agricultura, casa de campo, y pastoril. Alacant: Biblioteca Virtual Miguel de Cervantes. Disponible en http://www. cervantesvirtual.com/nd/ark:/59851/bmc416x2

Barros, C. (1997). La humanización de la naturaleza en la Edad Media. História, 19, 79-108.

Bleichmar, D., De Vos, P., Huffine, K., y Sheehan, K. (Eds.). (2009). Science in the Spanish and Portuguese Empires, 1500-1800. Stanford: Stanford University Press.

Castro, A. (2015). Plantas frutales. Cultura material en el reino de Chile. 1700 - 1850. Tesis Doctoral. Universidad Nacional de Cuyo.

Cordero Del Campillo, M., y Rojo Vázquez, F. (1979). La parasitología veterinaria en las obras de albeytería y afines. II. Fray Miguel Agustín s. XVII-XVIII. Revista Ibérica de Parasitología, 39, 209-221.

Denham, T. P., Iriarte, J., y Vrydaghs, L. (2016). Rethinking Agriculture: Archaeological and Ethnoarchaeological Perspectives. Londres y Nueva York: Routledge.

Descola, P. (2012). Más allá de naturaleza y cultura. Buenos Aires: Amorrortu.

Dillinger, T. L., Barriga, P., Escárcega, S., Jimenez, M., Lowe, D. S., y Grivetti, L. E. (2000). Food of the Gods: Cure for Humanity? A Cultural History of the Medicinal and Ritual Use of Chocolate. The Journal of nutrition, 130(8), 2057-2072.

Estienne, C. y J. Liebault (1689). L'Agriculture et maison rustique de maistres. París: Publicado por Claude Carteron y Charles Amy. Disponible en: http://memoirevive. besancon.fr/ark:/48565/a011290090127Ddfhvx/1/1

Ferigolo, J. (2014). A epistemología de Aristóteles. Rio Grande do Sul: Editora Unisinos.

Foucault, M. (1968). Las palabras y las cosas: una arqueología de las ciencias humanas. Buenos Aires: Siglo XXI.

Gately, I. (2003). Historia del tabaco. Barcelona: Ediciones B, SA.

Ginzburg, C. (1994). Mitos, emblemas, indicios. Morfología e historia. Buenos Aires: Gedisa.

Hayward, J. A. (1989). Historia de la medicina. Buenos Aires: Fondo de Cultura Económica.

Hell, B. (2001). Cazadores Rabiosos El dominio del salvajismo en el noroeste de Europa. En: P. Descola, y G. Pálsson (Eds.), Naturaleza y sociedad: perspectivas antropológicas (pp. 237-252). Buenos Aires: Siglo XXI.

Herrera, A. (1619). Libro de agricultura general del campo. Domingo Gonçalez, mercader de libros. Universidad de Granada. Biblioteca universitaria. Fondo Antiguo de Universidades y Colecciones Singulares. Disponible en: http://hdl.handle.net/10481/36597

Ingold, T. (1993). The Temporality of the Landscape. World archaeology, 25(2), 152-174.

Ingold, T. (2000). The Perception of the Environment. Londres: Rougledge.

Jones, O., y Cloke, P. (2008). Non-human Agencies: Trees in Place and Time. En: C. Knappett, y L. Malafouris (Eds.), Material Agency (pp. 79-96). New York: Springer US.

Latour, B. (2008). Reensamblar lo social-una introducción a la teoría del actor-red. Buenos Aires: Manantial.

Leemhart, M. (1961) Do Kamo. La persona y el mito en el mundo melanesio. Buenos Aires: Eudeba.

Le Goff, J. (1986). Lo maravilloso y lo cotidiano en el occidente medieval. Barcelona: Gedisa.

Le Goff, J. (1999). La civilización del occidente medieval. Barcelona, Buenos Aires, México: Paidós.

Lema, V. (2014). Hacia una cartografía de la crianza: domesticidad y domesticación en comunidades andinas. Espaço Amerindio, 8(1), 59.

Levi, G. (1996). Sobre microhistoria. En: P. Burke (Ed.), Formas de hacer historia (pp. 119-143). Madrid: Alianza Universidad.

Mafferra, L. (2018). Arqueología de los paisajes forestales del norte de Mendoza. Oxford: BAR, International Series.

Marconetto, B., y Mafferra, L. (2016). Todos los fuegos el fuego. Discusión en torno a las categorías modernas en 
la interpretación de registros antracológicos en contextos prehispánicos y coloniales. Cadernos do LEPAARQ (UFPEL), 13(25), 459-483.

Matzkevich, H. (2015). La influencia de Abraham Cohen de Herrera en la Filosofía Natural del siglo XVII: su impronta en los Principia Philosophiae de Anne Conway. Sefarad, 75(2), 345-379.

Mintz, S. W. (1996). Dulzura y poder: el lugar del azúcar en la historia moderna. México: Siglo XXI.

Morgado García, A. (2011). La visión del mundo animal en la España del siglo XVII: El Bestiario de Covarrubias. Cuadernos de historia moderna, 36, 67-88.

Musselman, L. J. (2003). Reference to trees in the holy book. Unasylva, 213, 45-52.

Nash, R. (2014). Wilderness and the American Mind. New Haven: Yale University Press.

Nieto Olarte, M. (2009). Ciencia, imperio, modernidad y eurocentrismo: el mundo atlántico del siglo XVI y la comprensión del Nuevo Mundo. Historia Crítica, 39, 12-32.

Núñez, L. P. (2008). Ediciones e historia textual del Libro de los secretos de agricultura de Miguel Agustín. Butlletí de la Reial Acadèmia de Bones Lletres de Barcelona, 51, 199-223.

Orlob, G. B. (1971). History of Plant Pathology in the Middle Ages. Annual Review of Phytopathology 9, 7-20.

Ovalle, A. (1646). Histórica relación del Reino de Chile. Roma. Disponible en: http://www.memoriachilena.cl/ archivos2/pdfs/mc0012104.pdf

Pastoureau, M. (2008). El oso: Historia de un rey destronado. Madrid: Grupo Planeta (GBS).

Pastoureau, M. (2013). Una historia simbólica de la Edad Media occidental. Buenos Aires: Katz Editores.

Pastoureau, M. (2015). El cerdo: Historia de un primo mal querido. Buenos Aires: Confluencias.

Piñero, J. M. L. (1979). Ciencia y técnica en la sociedad española de los siglos XVI y XVII. Madrid: Labor universitaria.

Piñero, J. y Tomás, J. P. (1996). La influencia de Francisco Hernández (1512-1587) en la constitución de la botánica y la materia médica modernas. Cuadernos valencianos de historia de la medicina, Serie A Monografías. Valencia: Universitat de València.
Puerto Sarmiento, F. J. (2009). La ciencia en España, el modelo ilustrado de expedición científica y la expedición botánica de José Celestino Mutis. I. Monografías de la Real Academia Nacional de Farmacia, 26, 57-75.

Prance, G. y Nesbitt, M. (2005). The Cultural History of Plants. Londrés: Routledge.

Quirós García, M. (2015). El Libro de Agricultura de Gabriel Alonso de Herrera: un texto en busca de edición. Criticón, $123,105-131$.

Salavert Fabiani, V. L. (1995). La cultura científica y técnica en la España de los siglos XVI y XVII. Bulletin Hispanique, 97(1)1, 233-259.

Salisbury, J. (2012). The Beast Within: Animals in the Middle Ages. Londres: Routledge.

Sánchez Martínez, A. (2011). Ciencia ibérica y mundo atlántico. Dynamis, 31(1), 245-259.

Sánchez Martínez, A. (2014). La "Atlantización" de la ciencia ibérica: el mundo atlántico visto desde la historia de la temprana ciencia moderna. Anuario de Estudios Atlánticos, 60, 29-66.

Slater, J. (2010). Todos son hojas: literatura e historia natural en el barroco español. Madrid: Editorial CSICCSIC Press.

Serra i Clota, A. (2011). Análisis de la construcción del manso en Catalunya, del libro: Llibre dels secrets d'agricultura, casa rústica i pastoril, de M. Agustí (1617). En: S. Huerta, I. Gil Crespo, S. García y M. Taín (Eds.), Actas del Séptimo Congreso Nacional de Historia de la Construcción (Vol. 2, pp. 1339-1348). Madrid: Instituto Juan de Herrera.

Sodigne-Costes, G. (1991). Le coeur et le cuir, la moelle et I'écorce. En: B. Ribemont, et al. (Eds.), Le «cuer» au Moyen Âge: Réalité et Senefiance. Aix-en-Provence: Presses universitaires de Provence. Disponible en: http:// www.openedition.org/6540

Terrell, J. E., Hart, J., Barut, S., Cellinese, N., Curet, A., Denham, T., Kusimba, C., Latinis, K., Oka, R., Palka, J., Pohl, M., Pope, W., Haines, H., y Staller, J. (2003). Domesticated Landscapes: The Subsistence Ecology of Plant and Animal Domestication. Journal of Archaeological Method and Theory, 10(4), 323-368.

Viazzo, P. P. (2003). Introducción a la antropología histórica. Lima: Pontificia Universidad Católica del Perú.

Volcy, C. (2007). Historia de los conceptos de causa y enfermedad: paralelismo entre la Medicina y la Fitopatología. Iatreia, 20(4), 407-421. 
L. E. Mafferra | Revista del Museo de Antropología 12 (3): 93-104 | 2019 http://dx.doi.org/10.31048/1852.4826.v12.n3.21895

Yáñez y Girona, A. (1845). Lecciones de historia natural: Botánica (Vol. 2). Barcelona: Benito Espona y Blay.
Willis, R. J. (2007). The History of Allelopathy. Dordrecht: Springer Science \& Business Media. 